\title{
Neuropsychiatric Manifestation Screening among Systemic Lupus Erythematosus Patients in Hasan Sadikin General Hospital Bandung
}

\author{
Septian Dwi Putra', Mulya Nurmansyah Ardisasmita², Laniyati Hamijoyo
}

\begin{abstract}
${ }^{1}$ Faculty of Medicine, Universitas

Padjadjaran, Bandung, Indonesia

${ }^{2}$ Department of Public Health, Faculty of

Medicine, Universitas

Padjadjaran, Bandung,

Indonesia

${ }^{3}$ Department of Internal Medicine,

Faculty of Medicine,

Universitas

Padjadjaran/Hasan

Sadikin General

Hospital, Bandung,

Indonesia
\end{abstract}

Corespondence:

Septian Dwi Putra, MD

Faculty of Medicine,

Universitas

Padjadjaran,

Email:s.d.putra36@

gmail.com

\begin{abstract}
Background: Systemic Lupus Erythematosus (SLE) is an autoimmune diseases caused by deposition of immune complex with the involvement of various organ system and certain autoantibodies production. One of the most significant manifestation is neuropsychiatric symptoms, known as NPSLE (Neuropsychiatric Systemic Lupus Erythematosus). This study aims to portray the distribution of neuropsychiatric manifestation of SLE patients using a screening method in Hasan Sadikin General Hospital.

Method: An observative, descriptive categoric study, with consecutive sampling were done. Data were collected by spreading the questionnaire to subjects diagnosed as SLE who visiting Hasan Sadikin General Hospital during August-October 2016 and by tracing their medical records. Data analyzed included age, sex, education background, duration of lupus, diagnosis criteria, ongoing therapy, and neuropsychiatric manifestation.
\end{abstract}

Result: Samples volunteered in this study were 97 patients. Of them 94 patients (96.9\%) were women and 47 patients (48.5\%) were senior high school graduated. Most common diagnosis criteria found was positive ANA test (91.8\%). Most necessary ongoing therapy given was methylprednisolone (96.9\%). Common neuropsychiatric manifestations were mood disorders $(73,2 \%)$, headache $(57,7 \%)$, and mononeuropathy $(51,5 \%)$.

Conclusion: The common psychiatric, central nervous, and peripheral nervous manifestations were mood disorder, headache, and mononeuropathy, respectively.

Keywords: Neuropsychiatry, NPSLE Screening, Systhemic Lupus Erythematosus.

\section{Introduction}

Systemic Lupus Erythematosus (SLE) or widely known as lupus, is an autoimmune diseases caused by deposition of immune complex with involvement of various organ systems and specific autoantibodies production. ${ }^{1,2}$ In Asia, prevalence of lupus was estimated around 50 to 100 cases per 100.000 population. ${ }^{3}$ Female patients has accounted for $76.4 \%-95.4 \%$ of all recorded SLE patients. ${ }^{4}$
Involvement of organ systems of lupus includes musculoskeletal, hematologic, mucocutaneus, neurologic, cardiopulmonary, renal, and ocular organ systems. One of the most significant manifestation is neurologic symptoms, with or without psychiatric symptoms, widely known as Neuropsychiatric Systemic Lupus Erythematosus (NPSLE). ${ }^{1,5}$ According to American College of Rheumatology, NPLSE was classified based on central and peripheral nervous manifestation onto 19 case definitions. ${ }^{5}$ NPSLE is found on $12.2 \%$ $94.7 \%$ patients prior to the diagnosis, during the diagnosis, following the diagnosis, or even during undetected or quiescent period of disease. ${ }^{4}$

NPSLE is quite burdensome in terms of quality of life, psychological comorbidity, and even mortality. Lower life quality were found in SLE patients who have NPSLE, includes any neurological, psychological-emotional, or cognitive dysfunction, rather than SLE patients without NPSLE. Besides life quality, the presence of psychological disturbance. like depression or anxiety, were higher among NPSLE patients. ${ }^{6}$ Mortality rate was also worrisome, as NPSLE patients may have 10-fold higher mortality rate than other SLE patients.? Further, NPSLE patients also have higher risk of having neurologic complications in the future. ${ }^{8}$

Despite the alarming burden of NPSLE, diagnosing NPSLE among SLE patients is quite challenging for physician. Such diagnosis should be made by the combination of immunology, neurology, and psychiatric examination. ${ }^{8,9}$ Fortunately, despite the complex assesment and burden of NPSLE, an adequate treatment can prevent the progression of disease. ${ }^{8,10}$ Therefore, it is important to encourage physicians in recognizing NPSLE in daily clinical practice. ${ }^{6-10}$

In Indonesia, especially in Department of Internal Medicine, Hasan Sadikin General Hospital, data regarding the distribution of neuropsychiatric manifestation among SLE patients had not been available yet. Such data were needed for physicians to increase the awareness of NPSLE and to be able to treat them adequately. NPSLE screening 
could be done using a questionnaire, even though it is not recommended to be used as the sole diagnosis. ${ }^{11}$ This study aims to portray the distribution of NPSLE manifestation using a NPSLE screening quistionnaire.

\section{Method}

Study was conducted at Rheumatology clinic in dr. Hasan Sadikin General Hospital, by using categoric descriptive study design. Subject of the study were all SLE patients visited Hasan Sadikin General Hospital during August to October 2016. Inclusion criteria were: all SLE patients recorded in medical record of Dr. Hasan Sadikin General Hospital, and routinely underwent follow-up during the period of the study. Patients with incomplete medical record data, lacking desired criteria, and unwilling to be involved in this study would be excluded. Minimum samples needed were 97 samples. Samples were selected by consecutive sampling method, which data collected until the minimum sample obtained.

NPSLE manifestation was screened by questionnaire. Questionnaire used was Mosca and colleague 38-questions 2011 questionnaire, and Kundu and colleague 2012 peripheral nervous sign of SLE. ${ }^{11,12}$ Questionnaire was translated with double translate method (English-Bahasa Indonesia-English) by certified translator, validated by Cronbach's Alpha of 0.802 and Pearson correlation value of 0.913 and 0.916 , respectively. Questionnaire was then consulted with Department of Neurology, Dr. Hasan Sadikin General Hospital. The questionnaires were filled by patients with supervision to minimize the bias. Questionnaire filling was done in workdays within time period of study until minimum sample obtained.

Secondary data were obtained from medical records, included age, sex, education background, duration of diseases, diagnosis criteria, and ongoing therapy. The collected data were sorted by variables and analyzed.

Ethical clearance were approved prior to data collection.

Analysis were conducted in descriptive manner by counting number and percentage by using Microsoft Office Excel. Variables of this study were age, sex, education background, duration of lupus, diagnosis criteria, ongoing therapy, and neuropsychiatric manifestation. Normality test of numeric variables data (age, age during the establishment of diagnosis, and duration of disease) was done using Saphiro-Wilk test to reveal whether the data is well distributed or not. Data were presented as mean " $( \pm$ Standard Deviation $)$ " if distributed well, or presented as "median (range)" if not distributed well.

\section{Results}

Within time period of the study, we were able to obtain minimum sample of 97 patients. Demographic data of the participants of the study were presented in Table 1 .
Table 1. Demographic Data

\begin{tabular}{lc}
\hline \multicolumn{1}{c}{ Characteristic (N=97) } & Results \\
\hline Age (years) & $33(14-61)^{*}$ \\
Women, N(\%) & $94(96.9)$ \\
Age of diagnosis (years) & $27(9-52)^{*}$ \\
Duration of Disease (years) & $4(0-20)^{*}$ \\
Educational Background, N(\%) & \\
$\quad$ Primary School & $11(11.3)$ \\
$\quad$ Junior High School & $20(20.6)$ \\
$\quad$ Senior High School & $47(48.5)$ \\
University & $19(19.6)$ \\
\hline
\end{tabular}

*: not normally distributed data, presented in median (range)

Duration of SLE were defined as age range since establishment of diagnosis until the time of this study conducted. Among 97 patients, most of participants were women $(96.9 \%)$ and graduated from senior high school (48.5\%).

Table 2. Distribution of SLE Patients According to Diagnosis Criteria and Ongoing Therapy

\begin{tabular}{|c|c|}
\hline $\begin{array}{l}\text { Distribution of patients } \\
N=97\end{array}$ & $\begin{array}{c}\text { Frequency } \\
\mathbf{N}(\%)\end{array}$ \\
\hline \multicolumn{2}{|l|}{ Diagnosis Criteria } \\
\hline Malar Rash & $64(66,0 \%)$ \\
\hline Discoid Rash & $37(38,1 \%)$ \\
\hline Photosensitivity & $65(67,0 \%)$ \\
\hline Oral Ulcers & $40(41,2 \%)$ \\
\hline Arthritis & $88(90,7 \%)$ \\
\hline Serositis & $23(23,7 \%)$ \\
\hline Renal Disorder & $26(26,8 \%)$ \\
\hline Neurology Disorder & $10(10,3 \%)$ \\
\hline Hematology Disorder & $45(46,4 \%)$ \\
\hline Immunology Disorder & $33(34,0 \%)$ \\
\hline Positive ANA Test & $89(91,8 \%)$ \\
\hline \multicolumn{2}{|l|}{ Ongoing Therapy } \\
\hline Cyclophosphamide & $11(11,3 \%)$ \\
\hline Methylprednisolone & $94(96,9 \%)$ \\
\hline Dexamethasone & $1(1,0 \%)$ \\
\hline Chloroquine & $45(46,4 \%)$ \\
\hline Azatioprin & $14(14,4 \%)$ \\
\hline Methotrexate & $5(5,2 \%)$ \\
\hline Cyclosporin A & $1(1,0 \%)$ \\
\hline Micophenolat Mophetyl & $0(0 \%)$ \\
\hline
\end{tabular}

Distribution of diagnosis criteria met on the first lupus diagnosis establishment were presented in Table 2, with positive ANA test (91.8\%) and arthritis (90.7\%) as the most common finding. Table 2 also showed ongoing therapy among participants was corticosteroid in form of methylprednisolone $(96,3 \%)$, while the other therapies were less frequent. 
Figure 1. Neuropsychiatric Manifestation Distribution Graphic

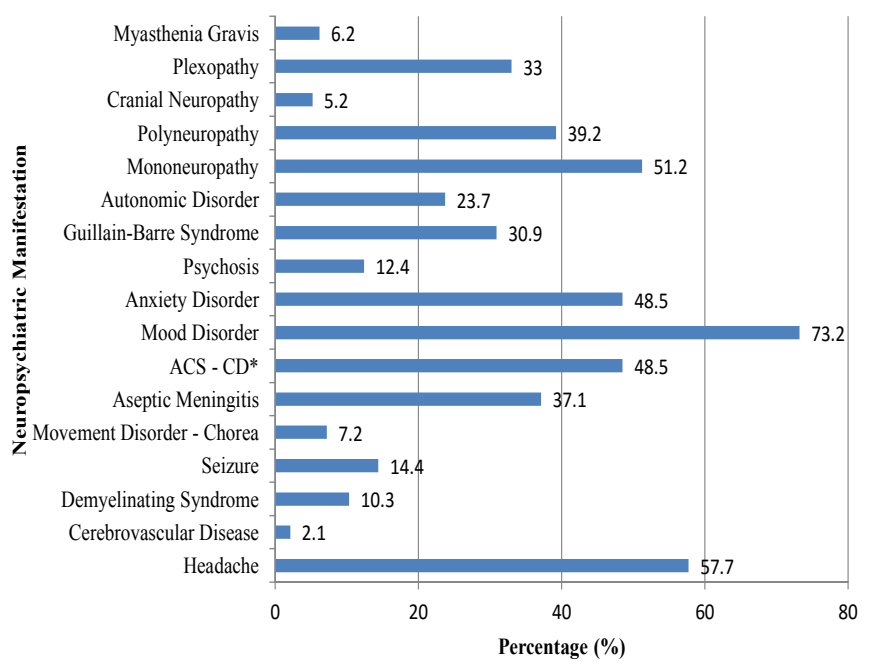

ACS - CD: Acute Confusional State, Cognitive Dysfunction

Figure 1 shows the percentage of each neuropsychiatric manifestation among subjects. Headache, cerebrovascular disease, demyleinating syndrome, seizure, chorea, and aseptic meningitis are classified as central nervous system manifestation of NPSLE. Acute convusional state-cognitive dysfunction, mood disorder, anxiety disorder, and psychosis are classified as psychiatry manifestation of NPSLE. GuillainBarre Syndrome, mononeuropathy, polyneuropathy, cranial neuropathy, plexopathy, and myasthenia gravis are classified as peripheral nervous system manifestation of NPSLE. The least common NPSLE findings was cerebrovascular disease (2.1\%), while the most common NPSLE complain is mood disorder $(73.2 \%)$.

\section{Discussion}

Lupus are likely to be occured in productive-aged women, ${ }^{4}$ same as our study's results which found $96,9 \%$ female participants with median age was 33 (14-61) years old. The similar finding also showed by the study conducted by Chiewthanakul, et al, which found the mean age SLE patients was $35 \pm 11.7$ years and Kampylafka et al, who found the mean age of SLE patients was $32 \pm 14$ years. ${ }^{13,14}$ Data of current age and age of SLE onset were collected due to fact that disease activity and damage were presented more in older age subjects. ${ }^{15}$ It shows that high level of disease activity conveyed by the level of interferon-alpha confirmed the conclusion that disease are more common in productive age. ${ }^{16}$ Median of duration of disease in this study was 4 years, with range between 0-20 years. It is similar with founding conducted by Kampylafka et $\mathrm{al}$, in which means duration of disease suffered by patients was $9 \pm 7.8$ years. ${ }^{13}$ There are no other NPSLE studies describing educational background of subjects. Educational background depicted person's perception of illness and often associated with NPSLE, especially cognitive function. ${ }^{17,18}$

Positive ANA test and arthritis were two most common criteria found in this study, while positive ANA test is the most common diagnosis criteria with percentage of $96 \%$ from all participants. The results were similar with Chiewthanakul, et al study. ${ }^{13}$

The use of therapy other than methylprednisolone such as cyclophosphamid, azatriopine, chloroquine, and methrotexate was rarely found. It was different compared with Chiewthanakul, et al study which presented wider variation of therapy with proportion of azatriopin, choloroquine, metothrexate, cyclophosphamide, and micophenolate mophetil were as follow $35 \%, 62 \%, 17 \%, 33 \%$, and $32 \%{ }^{13}$ It might be caused by the subject selection method in our study. We chose respondents in outpatient setting who were relatively in stable condition. Besides, not all drugs were available in Hasan Sadikin General Hospital pharmacy. Current therapy might affect direct or inderectly as secondary cause to NPSLE, by means of secondary infection caused by suppressed immunity or drug adverse effect. ${ }^{8,10}$

Central nervous system's manifestations in this study were dominated by headache. The finding is similar with the research conducted by Al-Shareef, et al which revealed headache in $34 \%$ subjects $;{ }^{19}$ and the research conducted by Abdel-Nasser, et al found headache prevalence was as much as $46.9 \%{ }^{20}$ Headache in SLE could be caused by many pathomechanism, but some studies suggested that headache-related SLE were caused by idiopathic intracranial hypertension. ${ }^{21}$

Cerebrovascular diseases was the least common finding in this study $(2.1 \%)$. The result was different with the finding in Al-Shareef, et al study, in which found the incidence of cerebrovascular in 35\% subjects. ${ }^{19}$ The difference is suggested due to the different modalities used in our study. The questionnaire can only confirm the preexisting complaint, but can not detect the cerebrovascular events as the standard examination; while in Al-Shareef, et al study every patients was assessed objectively by standard examination in hospital. ${ }^{19}$ Despite the difference, detecting cerebrovascular events in SLE patients is important because SLE patients reported having 2.05 fold higher chances to be hospitalized from cerebrovascular events. ${ }^{22}$

The finding of aseptic meningitis in our study was quite high (37.1\%). It is different with the result of study conducted by Al-Shareef et al, which only revealed $1.4 \%$ cases among their study subjects. ${ }^{19}$ The difference might be happened due to over presumptive of aseptic meningitis by the screening from questionnaire. The true diagnosis of aseptic meningitis is needed to be investigated further. Nevertheless, with a high result of aseptic meningitis presumption, physician should be aware of aspetic meningitis as to prevent complication such as neurological deficit and mortality in the future. ${ }^{23}$

Questionnaires in this study combined manifestations of acute confusional state and cognitive disfunction which has never conducted in any other study. Unseparated rate from both manifestations was $48.5 \%$. In studies conducted by Hanly, et al, Steaup Beekman, et al, and Morrison, et al, percentage of acute confusional state were tent to be low 
(2.6\%-6.9\%). Cognitive dysfunction was considerably high in the study conducted by Steup-Beekman, et al, with percentage of $26.5 \%{ }^{24-26}$ However, mood disorder was neccesary found in study conducted by Hanly, et al, with proportion of $16.5 \%$ from overall samples. ${ }^{26}$ The findings of mood disorders in this study were consistent with the study conducted by AbdelNasser, et al, with proportion of mood disorder as much as $59.4 \%$ of respondents, while we found $73.2 \%$ of respondents. ${ }^{20}$ Depression, the most common mood disorder in NPSLE, is a main concern for clinician treating SLE patients. Depression is not only a mere clinical manifestation, but also a serious threat to medical adherence, whereas SLE needs a long-term therapy. ${ }^{27}$

In peripheral nervous manifestations, the prevalence found were comparatively high compared to central nervous manifestation, included Guillan-Barre syndrome, otonomic nervous disorder, mononeuropathy, polyneuropathy, and plexopathy. The founding is different compared to the study perfomed by Hanly, et al and Morrison, et al. They found peripheral nervous system manifestation ranging between $0-24 \%$ and $0-7.9 \%$, respectively. ${ }^{26}$

Distribution of psychiatric disorder among lupus patients may vary, due to numerous factor, such as active autoantibodies and the method of diagnosis. ${ }^{28}$ We acknowledged our study were tend to be more subjective in making diagnosis because we only used questionnaire for assessing NPSLE manifestation. Exact diagnosis must be established by various supporting modalities, such as electroencephalogram, MRI, CT-scan, mental status examination, comprehensive nervous system examination, and other psychiatric status examination. ${ }^{9,28}$ Thus, several acquired data were not in line with other studies due to the different of interpretation and patients' subjectivity. Questionnaire was only describing 17 from 19 neuropsychiatric manifestation according to American College of Rheumatology (ACR) criteria since this questionnaire combined acute confusional state and cognitive disorder; and there were no questions to diagnose probable myelopathy. Uncompleted patients' medical record were also limiting this study.

\section{Conclusion}

The most common psychiatric manifestation was mood disorder. The most common CNS manifestation was headache. The most common peripheral nervous manifestation was mononeuropathy. Neuropsychiatry manifestation screening is recommended to be done routinely to all SLE patients. Further examination is needed when NPSLE manifestation found by the screening, so that optimal therapy could be given to the patients. Screening questionnaire used in this study still have to be tested to be used as a screening modality to detect neuropsychiatric manifestation.

\section{Reference}

1. Wallace DJ, Hahn BH. Definition and classification of lupus and lupusrelated disorders. In: Yazdany J, Dall'Era M, editors. Dubois' Lupus Erythematosus and Related Syndromes. 8th ed. Philadelphia: Elsevier Saunders; 2013. p. 1-7.

2. Longo D, Fauci A, Kasper D, et al. Systemic lupus erythematosus. In: Fauci AS, editor. Harrison's Principle of Internal Medicine. 18th ed. New York: McGraw-Hill; 2011. p. 2724-34.

3. Feng P. Systemic lupus erythematosus: the face of asia. Ann N Y Acad Sci. 2007;1108:114-20.

4. Unterman A, Nolte JES, Boaz M, et al. Neuropsychiatric syndromes in systemic lupus erythematosus: a meta-analysis. Semin Arthritis Rheum. Elsevier; 2011:41:1-11.

5. Liang MH, Corzillius $\mathrm{M}$, Bae SC, et al. The american college of rheumatology nomenclature and case definitions for neuropsychiatric lupus syndromes. Arthritis Rheum. 1999;42(4):599-608.

6. Pettersson S, Lövgren M, Eriksson LE, et al. An exploration of patientreported symptoms in systemic lupus erythematosus and the relationship to health-related quality of life. Scand J Rheumatol. 2012;41:383-90.

7. Zirkzee EJM, Huizinga TWJ, Bollen ELEM, Buchem MA Van, Middelkoop HAM. Mortality in neuropsychiatric systemic lupus erythematosus (NPSLE). Lupus. 2014;23:31-8.

8. Joseph FG, Scolding NJ. Neurolupus. Pract Neurol. 2010;10:4-15.

9. Hanly JG. Diagnosis and management of neuropsychiatric SLE. Nat Rev Rheumatol. Nature Publishing Group; 2014;15:1-10.

10. Wallace DJ, Hahn BH. Clinical Aspects of the Nervous System. In: West SG, editor. Dubois' Lupus Erythematosus and Related Syndromes. 8th ed. Philadelphia: Elsevier Saunders; 2013. p. 368-81.

11. Mosca M, Govoni M, Tomietto $P$, et al. The development of a simple questionnaire to screen patients with SLE for the presence of neuropsychiatric symptoms in routine clinical practice. Lupus. 2011;20:485-92.

12. Kundu AK, Maity A. Neuropsychiatric lupus. Med Updat. 2012;22:64652.

13. Kampylafka El, Alexopoulos H, Kosmidis ML, et al. Incidence and prevalence of major central nervous system involvement in systemic lupus erythematosus : a 3 -year prospective study of 370 patients. PLoS One. 2013;8(2):1-8.

14. Chiewthanakul P, Sawanyawisuth K, Foocharoen C, Tiamkao S. Clinical features and predictive factors in neuropsychiatric lupus. Asian Pacific $J$ Allergy Immunol. 2012;30(1):55-60.

15. Lalani S, Pope J, Leon F De, Peschken C. Clinical features and prognosis of late-onset systemic lupus erythematosus: results from the 1000 faces of lupus study. J Rheumatol. 2010;37(1):38-44.

16. Niewold TB, Adler JE, Glenn SB, et al. Age- and sex-related patterns of serum interferon-alpha activity in lupus families. Arthritis Rheum. 2008;58(7):2113-9.

17. Croog SH. Ethnic origins, educational level, and responses to a health questionnaire. Hum Organ. 1961;20(2):65-9.

18. Conti F, Alessandri C, Perricone $C$, et al. Neurocognitive dysfunction in systemic lupus erythematosus: association with antiphospholipid antibodies, disease activity and chronic damage. PLoS One. 2012;7(3):1-7.

19. Al-shareef A, Attar SM. Central nervous system manifestation in patients with SLE: a 12-year retrospective chart review at a tertiary center. Kuwait Med J. 2014;46(1):14-20.

20. Abdel-Nasser AM, Ghaleb RM, Mahmoud JA, Khairy W, Mahmoud RM. Association of anti-ribosomal P protein antibodies with neuropsychiatric and other manifestations of systemic lupus erythematosus. Clin Rheumatol. 2008;27:1377-85.

21. Kim J-M, Kwok S, Ju JH, Kim H, Park S. Idiopathic intracranial hypertension as a significant cause of intractable headache in patients with systemic lupus erythematosus: a 15-year experience. Lupus. 2012;21:542-7. 
22. Ward MM. Premature morbidity from cardiovascular and cerebrovascular diseases in women with systemic lupus erythematosus. Arthritis Rheum. 1999;42(2):338-46.

23. Baizabal-Carvallo JF, Delgadillo-Márquez G, García-Ramos G, Estañol B. Clinical characteristics and outcomes of the meningitides in systemic lupus erythematosus. Eur Neurol. 2009;61:143-8.

24. Steup-Beekman GM, Zirkzee EJM, Cohen D, et al. Neuropsychiatric manifestations in patients with systemic lupus erythematosus: epidemiology and radiology pointing to an immune-mediated cause. Ann Rheum Dis. 2013;72(2):1176-9.
25. Morrison E, Carpentier S, Shaw E, Doucette S, Hanly J. Neuropsychiatric systemic lupus erythematosus: association with global disease activity. Lupus. 2014;23(4):370-7.

26. Hanly JG, Urowitz MB, Su L, et al. Prospective analysis of neuropsychiatric events in an international disease inception cohort of patients with systemic lupus erythematosus. Ann Rheum Dis. 2010;69(3):529-35.

27. Julian LJ, Yelin E, Yazdany J, et al. Depression, medication adherence, and service utilization in systemic lupus erythematosus. Arthritis Rheum. 2009;61(2):240-6.

28. Stojanovich L, Zandman-goddard G, Pavlovich S, Sikanich N. Psychiatric manifestations in systemic lupus erythematosus. Autoimmun Rev. 2007;6:421-6. 